\title{
Fibroma osificante central en el maxilar superior: reporte de un caso y revisión de la literatura
}

\section{Central ossifying fibroma in the maxilla: a case report and review of the literature}

\author{
Chrcanovic BR*, López Alvarenga R**, Horta MCR***, Freire-Maia B****, \\ Souza LN*****
}

\section{RESUMEN}

El fibroma osificante central (fibroma cementificante / fibroma cemento osificante) es un tumor benigno óseo bien delimitado, se localiza principalmente en los maxilares aunque se puede presentar en los huesos orbital, frontal, paranasal y temporal. Se caracteriza por presentar un crecimiento y una tumefacción deformante de evolución lenta con una incidencia mayor en la mandíbula. El tratamiento consiste en la extirpación quirúrgica de la lesión. En este artículo se presenta un caso de fibroma osificante central localizado en la región posterior derecha del maxilar superior, de gran dimensión, que acomete a una paciente de sexo femenino de 46 años de edad, revelando datos clínicos, aspectos diagnósticos, imagenológicos, histopatológicos así como su tratamiento.

Palabras clave: Neoplasia benigna, Fibroma osificante, Lesiones fibroóseas, Seno maxilar.

\section{SUMMARY}

Central ossifying fibroma (cementing fibroma / cemento ossifying fibroma) is a bone benign tumor almost exclusively from maxillary region but can be in the orbital bones, frontal, paranasal and temporal. Progressive growth and disfiguring swelling of slow evolution are characteristics with more prevalence in the mandible. The treatment choice is a surgical excision of the lesion. This article presents a case of central ossifying fibroma located in the region of the right posterior maxilla of large dimension of a female patient of 46 years of age, describing clinical, diagnostic, imaging, and histopathological aspects as well as the treatment employed.

Key words: Benign neoplasm, Ossifying fibroma, Fibro-osseous lesions, Maxillary sinus.

Fecha de recepción: 12 de agosto de 2009.

Aceptado para publicación: 2 de octubre de 2009.

Chrcanovic BR, López Alvarenga R, Horta MCR, Freire-Maia B, Souza LN. Fibroma osificante central en el maxilar superior: reporte de un caso y revisión de la literatura. Av. Odontoestomatol 2011; 27 (1): 33-39.

* Departamento del Servicio de Cirugía y Traumatología Buco Maxilofacial del Hospital Maria Amélia Lins (HMAL) de Belo Horizonte, Brasil.

** Licenciado en Odontología por la universidad del Centro Universitario Newton Paiva, Belo Horizonte, Brasil.

*** Prof. Adjunto de Patología Bucal, Facultad de Odontología da PUC Minas (Pontifícia Universidade Católica), Belo Horizonte, Brasil.

**** Prof. Asistente de Cirugía Buco Maxilofacial, Facultad de Odontología da PUC Minas (Pontifícia Universidade Católica), Belo Horizonte, Brasil.

***** Prof. Adjunto de Cirugía Buco maxilofacial, Centro Universitario Newton Paiva, Belo Horizonte, Brasil. 


\section{INTRODUCCIÓN}

El fibroma osificante central (FOC) es un tumor benigno óseo, el cual se incluye dentro de las llamadas lesiones fibroóseas, que se caracterizan por el reemplazo de la arquitectura ósea normal por fibroblastos, tejido fibroso y cantidades variables de tejido mineralizado $(1,2)$.

Se manifiesta con más frecuencia en la mandíbula que en el maxilar superior, localizándose preferentemente en la región molar (52\%) o premolar (25\%). Es más comúnmente diagnosticada en mujeres (5:1) entre la $3^{\mathrm{a}}$ y $4^{\mathrm{a}}$ década de la vida (1-5).

Clínicamente las lesiones pequeñas cursan con la ausencia de sintomatología y en las lesiones de mayor tamaño, se evidencia el aumento de volumen con asimetría facial, de crecimiento lento, consistencia dura e indolora $(1,2)$.

En los exámenes radiográficos el FOC se presenta como áreas radiolúcidas o mixtas en distintos grados, unilocular o multilocular bien delimitada, expansión de corticales óseas y ocasional desplazamiento dentario y/o reabsorción radicular $(1,2,4)$.

Histológicamente se constituye de una cápsula fibrosa ocasional, trabéculas óseas, masas cementarias redondeadas u ovoideas, celularidad variable con fibras densas, presencia de células gigantes y hemorragia reciente (1-4).

El tratamiento del FOC incluye la extirpación quirúrgica y legrado del lecho óseo con una extensión que depende del tamaño y de la localización de la lesión $(1,2,6)$.

El objetivo de este trabajo es presentar un caso de FOC localizado en la región posterior derecha del maxilar superior, de gran extensión, en el cual se discuten los aspectos característicos del caso y hacer una breve revisión de la literatura.

\section{CASO CLÍNICO}

Paciente femenino de 46 años de edad, sin antecedentes patológicos de interés, fue remitido a la Clíni- ca de Medicina Oral de la Facultad de Odontología de la PUC-Minas (Pontifícia Universidade Católica) por su odontólogo, por presentar una tumoración asintomática en la zona posterior del maxilar superior del lado derecho con aproximadamente 1 año de evolución.

En el examen extraoral se evidenció una asimetría facial provocada por el aumento de volumen del maxilar superior del lado derecho (Fig. 1a).

Durante la exploración intraoral se observó un abombamiento indoloro del paladar duro y cortical vestibular del maxilar superior del lado derecho correspondiente al espacio edéntulo de los elementos dentarios 1.5, 1.6, 1.7, 1.8 de aproximadamente 3$4 \mathrm{~cm}$ de diámetro y de consistencia dura con mucosa de características normales al tejido adyacente (Figs. 1b y c).

Los exámenes radiográficos (Panorámica, Periapicales y Oclusal) revelaron una imagen circunscrita radiolúcida, unilocular con focos radioopacos, presentándose desde el canino superior (1.3) hasta la región de tuberosidad maxilar y reabsorción radicular en los dientes 1.3 y 1.4 (Figs. 2a, b y c) . En la tomografía computarizada (TAC) se observó claramente la alteración de la arquitectura ósea, con una lesión de intensidad mixta, delimitada por un halo hiperdenso

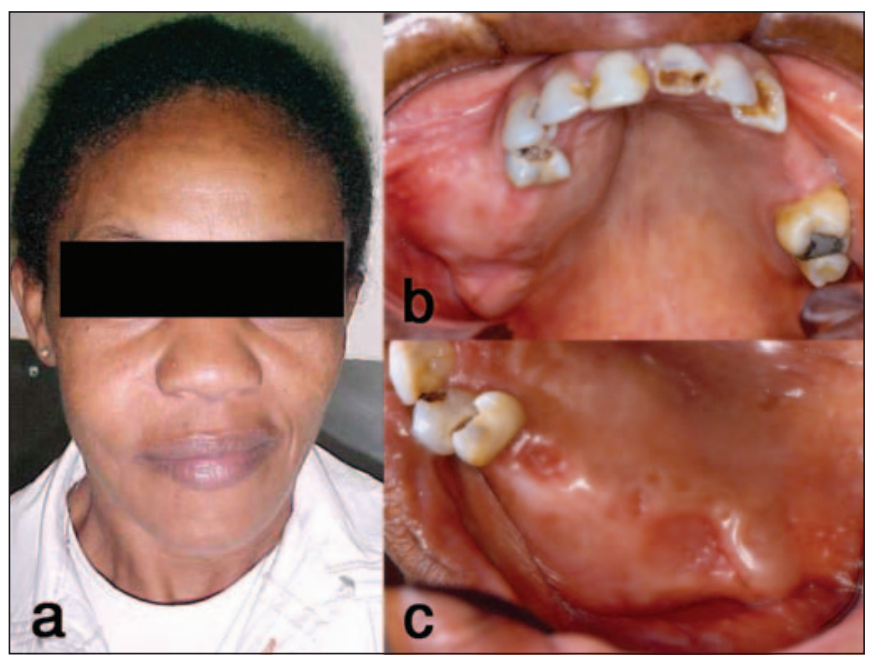

Fig. 1. a. Asimetría facial. b y c. Visión directa intraoral del aumento de volumen del paladar duro y reborde alveolar localizada en maxilar superior del lado derecho correspondiente al espacio edéntulo. 
involucrando al seno maxilar y desplazando la cortical vestibular, así como un desplazamiento y rompimiento de la pared lateral de fosa nasal y del suelo orbitario ambos del lado derecho (Figs. 2d y e). La vitalidad pulpar de los dientes involucrados fue positiva.

Se realizó una biopsia por punción aspiración en la cual no se obtuvo ningún contenido líquido. Bajo anestesia local se procedió a realizar la biopsia incisional para el análisis anatomopatológico. Los cortes histológicos coloreados con hematoxilina-eosina mostraron un tejido conjuntivo fibroso, el cual presentaba varios grados de celularidad con contenido de material mineralizado. La porción de tejido duro se encontraba de forma trabecular, osteoide, hueso o esferas celulares basofílicas que son similares al cemento. La lesión se presentaba separada de la fina cortical ósea por un tejido conjuntivo fibroso (Figs. 3a, b y c). Los hallazgos fueron compatibles con una lesión fibroósea benigna. Basado en las características clínicas, radiográficas y el examen anatomopatológico se estableció el diagnostico de fibroma osificante central.

Bajo el diagnóstico de FOC el paciente fue derivado al Servicio de Cirugía y Traumatología Buco maxilo- facial del Hospital Maria Amélia Lins (HMAL) para tratamiento. El paciente fue intervenido quirúrgicamente bajo anestesia general, se realizó enucleación completa de la tumoración, legrado del lecho óseo y avulsión del elemento dentario 1.4 (Figs . 4a y b). Se remitió el material enucleado en la cirugía para análisis anatomopatológico (Figs. 5a y b), en el cual se confirmó el diagnóstico de fibroma osificante central.

El seguimiento del paciente con controles periódicos no mostró en 2 años signos de evidencia de recidiva.

\section{DISCUSIÓN}

El fibroma osificante fue descrito por Menzel en 1872 y en 1927 el término fibroma osificante fue utilizado por primera vez por Montgomery, nombre por el cual actualmente se reconoce a la lesión $(7,8)$. Hasta 1948 se pensó que la displasia fibrosa y el fibroma osificante eran la misma enfermedad o que una era la variante de la otra (9). A comienzos de los años cincuenta Sherman y Sternberg con sus estudios detallados de los aspectos clínicos, radiológicos, his-
Fig. 2. a. Radiografía oclusal superior, donde se aprecia los limites lateral, medial y anterior de la lesión, así como reabsorción radicular en los dientes 1.3 y 1.4 . b. Radiografía panorámica reveló que la lesión se extendía desde el canino superior (1.3) hasta la región de tuberosidad maxilar y hacia el seno maxilar. c. Radiografia periapical mostrando imagen circunscrita radiolúcida, unilocular con focos radioopacos y reabsorción radicular en el diente 1.4. d y e. Tomografía computarizada - cortes coronal y axial, revelando la extensión de la lesión.

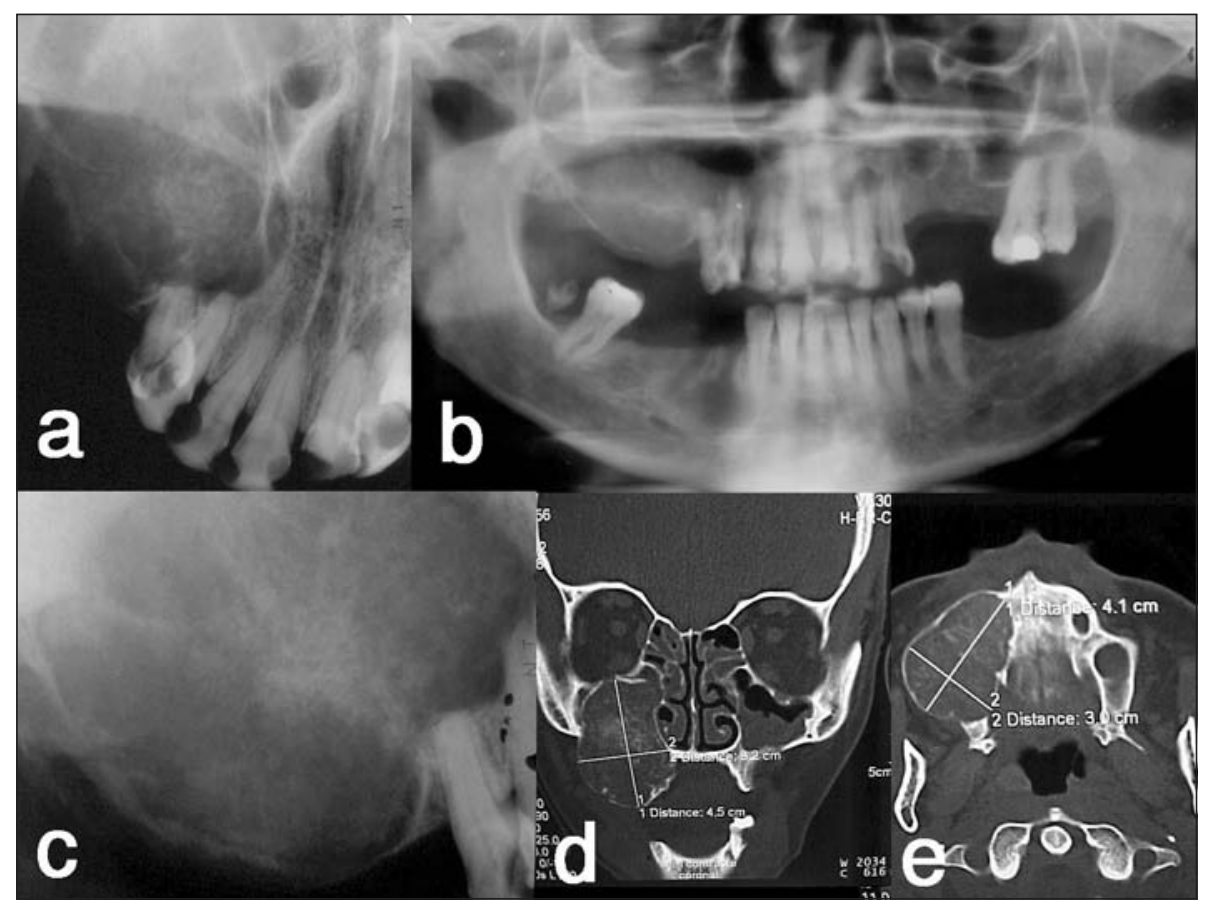




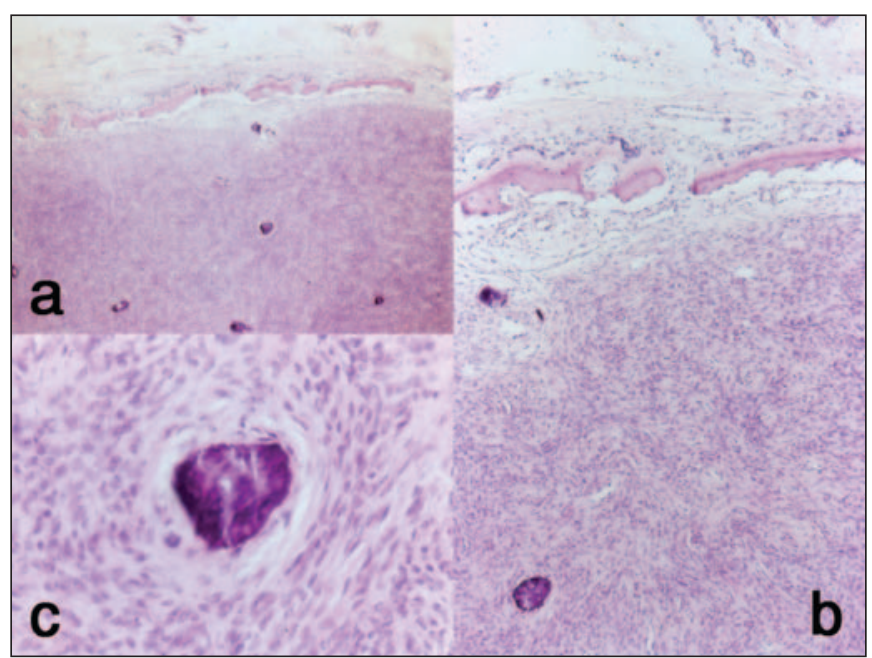

Fig. 3. Estudio anatomopatológico - (HE 50x), (HE 100x), (HE 400x).



Fig. 4. a. Imagen intraoperatorio, donde se observa la enucleación completa de la lesión (la lesión se separó fácilmente del hueso subyacente). b. Aspectos de la cavidad ósea tras la enucleación.

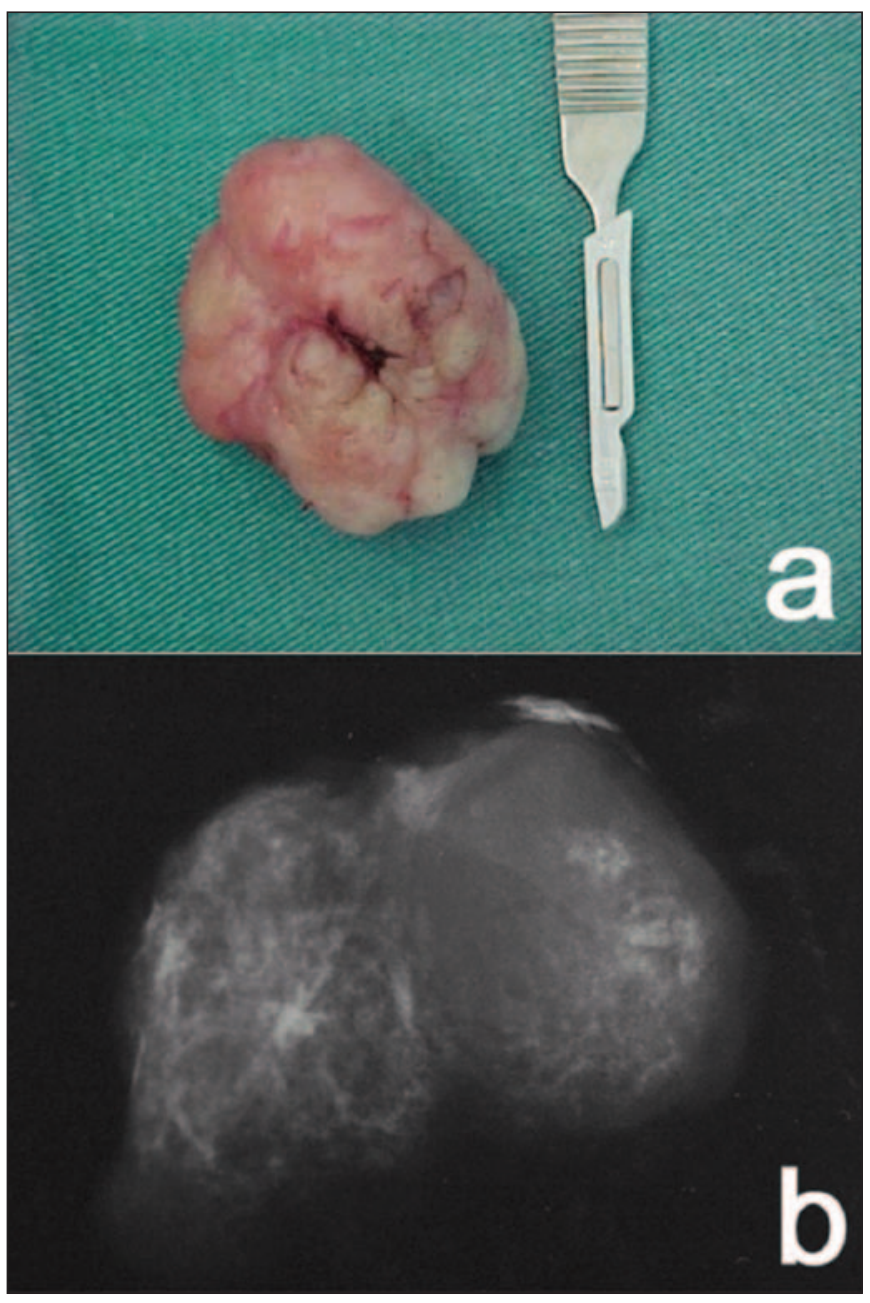

Fig. 5. a. Espécimen quirúrgico, se observa una lesión fibrosa, encapsulada, no friable, de consistencia dura a la palpación, no sangrante y con un tamaño aproximado de 4,5 x 4,1 x 3,2 cm. b. Radiografía de la lesión hecha fuera de la cavidad oral donde se aprecia focos radioopacos (zonas mineralizadas).

tológicos y patológicos, dividieron estás, en dos identidades diferentes $(4,10)$.

El nombre fibroma cemento osificante fue adoptado desde el comienzo de la década de los 90 por la OMS, es considerado actualmente una neoplasia fibroósea y está englobado dentro de los tumores no odontogénicos $(3,11)$.

El FOC, aunque sea una lesión poco frecuente, es probablemente la lesión más común dentro de las neoplasias fibroóseas, que se origina a partir de células del ligamento periodontal $(3,12)$. 
Radiológicamente el FOC se presenta como áreas radiolúcidas o mixtas en distintos grados. Se manifiesta como una lesión unilocular o multilocular bien delimitada, en cuyo interior podemos encontrar cantidades variables de material radiopaco. Pudiendo también presentar reabsorción radicular y desplazamiento de raíces vecinas $(1,2,4)$. En el caso presentado los hallazgos radiográficos fueron compatibles con los descritos en la literatura.

Los fibromas osificantes se clasifican de acuerdo con el tipo de tejido que se encuentre, si predomina el hueso se considera fibroma osificante, si existe presencia de trabéculas curvilíneas o calcificaciones esféricas se considera fibroma cementificante y si se observa tejido óseo y cemento se denomina fibroma cemento osificante. Algunos autores no encontraron diferencias histológicas entre el fibroma cemento osificante y el fibroma osificante (4). La nomenclatura actual se simplifica refiriéndose a todas las lesiones de este grupo como fibromas osificantes $(4,13)$.

A nivel histopatológico, la literatura es clara en señalar que aún existe controversia en cuanto el diagnóstico para las lesiones fibroóseas. La displasia fibrosa y el fibroma osificante tienen gran similaridades histológicas, siendo la presencia de una cápsula fibrosa la única diferencia entre estas patologías, ya que este hallazgo es raro en el caso de la displasia fibrosa $(14,15)$. Algunos autores también señalan que la variación en los tipos de material mineralizado ayuda a distinguir el fibroma osificante de la displasia fibrosa, la cual tiene un patrón más uniforme de diferenciación ósea $(1,2,15)$. Varios autores están de acuerdo en que para obtener un diagnóstico apropiado y preciso de las lesiones fibroóseas es fundamental la exploración clínica, radiológica y quirúrgica (16).

El diagnóstico diferencial descrito en la literatura incluye otras entidades fibroóseas como la displasia óseo-cementaria (displasia focal periapical y florida), la displasia fibrosa, el osteoma osteoide, osteoblastoma y la osteomielitis crónica esclerosante $(2,3,11)$. Sin embargo, nunca hay que descartar otras lesiones radiotransparente mandibulares como el quiste óseo solitario, queratoquiste, ameloblastoma, granuloma de células gigantes, mixoma, tu- mor odontogénico adenomatoide, mieloma múltiple, osteosarcoma de bajo grado, cementoblastoma $(2,17,18)$.

Algunas características clínicas importantes, que indicaron el comportamiento benigno del tumor en este caso clínico, fueron el crecimiento lento y gradual de la lesión y la zona de aumento de volumen que se presentaba cubierta por una mucosa de características normales al tejido adyacente.

De los casos clínicos de fibroma osificante encontrados en la literatura existe un subtipo de fibroma llamado "fibroma osificante juvenil" que es una variante de mayor agresividad y de crecimiento rápido, que se presenta en niños entre 5 y 15 años de edad (20). Se manifiesta con más frecuencia en el maxilar superior que en la mandíbula, aunque se localiza principalmente en los huesos orbitales, frontales y paranasales (20). En el caso presentado sólo coincidió en la localización, ya que fue en el área maxilar, el resto no corresponde con las características de este tipo de fibroma.

El tratamiento más utilizado es la remoción quirúrgica de la lesión, pudiendo en este momento ser realizada un curetaje. Se recomienda la resección en bloque en los casos en que se presente recurrencia después de la primera intervención mediante enucleación de la lesión o en los casos que la lesión sea extensa y agresiva $(3,4,6,19)$. El protocolo utilizado para el tratamiento de este caso particular fue la enucleación completa de la lesión y curetaje del lecho óseo.

El pronóstico del FOC es excelente y las recurrencias son poco frecuentes. No ha sido documentado en la literatura ningún caso de malignización (3).

\section{CONCLUSIONES}

La revisión de la literatura y el caso clínico descrito permiten realizar las siguientes consideraciones:

- Los autores son bastantes uniformes en relación las características clínicas del FOC, relatando la predilección de la lesión por el sexo femenino entre la $3^{a}$ y $4^{a}$ década de la vida, más 
frecuente en la mandíbula con preferencia en el sector posterior y que evoluciona lentamente. Sin embargo, otras regiones pueden ser afectadas.

- El FOC es un tumor poco frecuente, pero es probablemente la lesión más común dentro de las neoplasias fibroóseas.

- Las lesiones fibroóseas pueden ser muy similares entre sí, siendo de suma importancia los exámenes clínicos, radiográficos, quirúrgicos e histológicos para determinar el correcto diagnóstico.

\section{BIBLIOGRAFÍA}

1. Su L, Weathers D, Waldron C. Distinguishing features of focal cemento-osseus and cementoossifying fibromas. Oral Surg Oral Med Oral Pathol. 1997;84:301-9.

2. Neville BW, Damm DD, Allen CM, Bouquot JE. Oral and Maxillofacial Pathology. 2nd ed. Filadelfia: Saunders. 2002;563-4.

3. Waldron C. Fibro-osseus lesions of the jaws. J Oral Maxillofac Surg. 1993;51:828-35.

4. Eversole LR, Leider AS, Nelson K. Ossifying fibroma: a clinicopathologic study of sixty-four cases. Oral Surg Oral Med Oral Pathol. 1985;60: 505-11.

5. Rosenberg A, Mokhtari H, Slootweg PJ. The natural course of an ossifying fibroma. Int J Oral Maxillofac Surg. 1999;28:454-6.

6. Hauser MS, Freije S, Payne RW, Timen S. Bilateral ossifying fibroma of the maxillary sinus. Oral Surg Oral Med Oral Pathol. 1989;68(6): 759-63.

7. Yih WY, Pederson GT, Bartley MH. Multiple familial ossifying fibromas: relationship to other osseous lesions of the jaws. Oral Surg Oral Med Oral Pathol. 1989;68:754-8.
8. Montgomery $\mathrm{AH}$. Ossifying fibroma of the jaw. Arch Surg 1927;15:30-44.

9. Huebner GR, Brenneise CV, Ballenger J. Central ossifying fibroma of the anterior maxilla: report of case. J Am Dent Assoc. 1988;116:507-10.

10. Sherman RS, Sternberg WC. Roentgen appearance of ossifying fibroma of bone. Radiology. 1948;50:295-309.

11. De Vicente Rodríguez JC, González Méndez S, Santamaría Zuazua J, Rubiales B. Tumores no odontogénicos de los maxilares: clasificación, clínica y diagnóstico. Med Oral. 1997;12: 83-93.

12. Mesquita RA, Sousa SCOM, Araújo NS. Proliferative activity in peripheral ossifying fibroma and ossifying fibroma. J Oral Pathol Med. 1998;27:64-7.

13. MacDonald-Jankowski DS. Cemento-ossifying fibromas in the jaws of Hong Kong Chinese. Dentomaxillofac Radiol. 1998;27:298-304.

14. Zachariades N, Vairaktaris E, Papanicolau S, Triantafyllou D, Papavassiliou D, Mezitis M. Ossifying fibroma of the jaws. Review of the literature and report of 16 cases. Int J Oral Surg. 1984;13:1-6.

15. Regezi JA, Derr DA, Courtney RM. Odontogenic tumors: analysis of 706 cases. J Oral Surg. 1978;36:771-8.

16. Sakuma T, Kawasaki T, Watanabe K. Concurrent Cementifying and Ossifying Fibromas of the Mandible: Report of a case. J Oral Maxillofac Surg. 1998;56:778-82.

17. Aguirre JM. Caballero R. Diagnóstico diferencial de las lesiones radiotrasparentes de los maxilares. Medicina Oral Barcelona: Masson SA. 1995;685-97.

18. Jham BC, Duraes GV, Rocha Santos CR. Extensive central ossifying fibroma of the maxilla: a case report with description of an alternative 
surgical technique. Minerva Stomatol. 2006 Oct;55(10):593-7.

19. Tamiolakis D, Thomaidis V, Tsamis I, Lambropoulou M, Alexiadis G, Seretis K, Papadopoulos N. Cementifying-Ossifying Fibroma Of The Maxilla: A Case Report. Acta Stomatol Croat. 2005;39(3):315-8.

20. Wiedenfeld K, Neville B, Hutchins A, Bell R, Brock T. Juvenile ossifying fibroma of the maxilla in a 6 year old male: case report. Ped Dent. 1995;17: 365-7.

\section{CORRESPONDENCIA}

Rodrigo López Alvarenga

Gomera, 6, esc. 2, $3^{\circ} \mathrm{H}$

04770 Adra. Almería

E-mail: mre_lopez@hotmail.com 
